\title{
Extending Hirshfeld-I to bulk and periodic materials
}

\author{
D. E. P. Vanpoucke,,$^{1,2}$ P. Bultinck, ${ }^{2}$ and I. Van Driessche ${ }^{1}$ \\ ${ }^{1}$ SCRiPTS group, Dept. Inorganic and Physical Chemistry, \\ Ghent University, Krijgslaan 281 - S3, 9000 Gent, Belgium \\ ${ }^{2}$ Ghent Quantum Chemistry Group, Dept. Inorganic and Physical Chemistry, \\ Ghent University, Krijgslaan 281 - S3, 9000 Gent, Belgium
}

(Dated: July 13, 2012)

\begin{abstract}
In this work, a method is described to extend the iterative Hirshfeld-I method, generally used for molecules, to periodic systems. The implementation makes use of precalculated pseudo-potential based electron density distributions, and it is shown that high quality results are obtained for both molecules and solids, such as ceria, diamond, and graphite. The use of grids containing (precalculated) electron densities makes the implementation independent of the solid state or quantum chemical code used for studying the system. The extension described here allows for easy calculation of atomic charges and charge transfer in periodic and bulk systems. The conceptual issue of obtaining reference densities for anions is discussed and the delocalization problem for anionic reference densities originating from the use of a plane wave basis set is identified and handled.
\end{abstract}

PACS numbers:

\section{INTRODUCTION}

One of the most successful concepts in chemistry is that of "atoms in molecules" (AIM). It states that the properties of a molecule can be seen as simple sums of the properties of its constituent atoms. An impressive amount of insights has been obtained from such a viewpoint, although a precise definition of an AIM remains elusive. ${ }^{1-3}$ All AIM methods have the common purpose of trying to improve our understanding of chemical concepts such as molecular similarity and transferability between molecules. ${ }^{4}$ Since the concept of AIM is basically about how one should divide the electrons, more specifically the electron density distribution (EDD) $\rho(\mathbf{r})$ in the molecule between the different "atoms", this leads to two obvious categories of approaches in which most of the methods used for defining AIM can be divided. The first category of approaches is based on the wave-function/states of the system, and most of the work is performed in the Hilbert space of the basis functions used. One of the most famous examples here is the Mulliken approach. ${ }^{5,6}$ The second category of approaches is based on the division of the EDD as it exists in real-space. In these real-space approaches the molecule is split into atomic basins, that can overlap such as in the Hirshfeld ${ }^{7}$ and derived methods, ${ }^{8-12}$ or that are non-overlapping such as in Bader's approach. ${ }^{13}$

The concept of AIM is strongly linked to the concept of transferability. Because both are central in chemistry, and chemists mainly focus on molecules, they are mostly used for molecules. ${ }^{14,15}$ There is, however, no reason why these concepts should not be applicable for periodic systems such as bulk materials. Even more, if these concepts are truly valid, they should hold equally well for solids as for molecules, and should provide additional insight in the chemical properties of defects, such as dopants, interfaces and adsorption of molecules on surfaces.
To this date we are aware of few implementations for periodic systems of the Hirshfeld and Hirshfeld-I approach, but their number is steadily rising. Martín Pendás et $a .^{16}$ investigated Hirshfeld surfaces as approximations for interatomic surfaces for $\mathrm{LiF}$ and $\mathrm{CS}_{2}$ crystals. The Cut3D plugin of the ABINIT code can be used to calculate Hirshfeld charges, ${ }^{17-20}$ and recently Leenaerts et al. implemented a "subsystem" based Hirshfeld-I method to study graphane, graphene fluoride and paramagnetic adsorbates on graphene. ${ }^{21-23}$ Similarly, Spackman and collaborators presented subsystem-based Hirshfeld surfaces for molecular solids and implemented this in their Crystal-Explorer code. $^{24-28}$ In a recent publication Watanabe et al. ${ }^{29}$ presented Hirshfeld results for metal-organic frameworks using the DDEC-code of Manz and Sholl. ${ }^{12}$ The same code was probably also used in the investigation of charge injection in graphene layers by Rogers and Liu. ${ }^{30}$ These authors present Hirshfeld-I charges, though they do not mention explicitly how these were obtained. In this DDEC code the function which is minimized is a linear combination of the function minimized in the Hirshfeld-I method, the one minimized in the iterative stockholder approach, and an additional term enforcing the constraint that all electron density is accounted for. $^{8-12}$ At the time of writing Verstraelen et al. showed that an excellent reproduction of the electrostatic potential (ESP) in silicates is possible using Hirshfeld-I charges. ${ }^{31}$

In this work, we have implemented an extension of the iterative Hirshfeld-I approach ${ }^{9}$ to periodic systems, as a module in our HIVE-code. ${ }^{32}$ The implementation makes use of grid stored EDDs, which can easily be generated by standard solid state and quantum chemical codes.

In Sec. II a short review of the parameters used in the solid state and quantum chemical codes is given. Afterwards the basic theory behind the Hirshfeld-I method is presented and extended to periodic systems. In 
addition, the spatial integration grid, pseudo-potentials and stored EDDs are discussed in view of the Hirshfeld-I method for periodic systems. In Sec. III the influence of the different grids on the accuracy is discussed. We also identify a delocalization problem in the radial EDDs which originates from the plane wave approach and periodic boundary conditions (PBC) used in the solid state code. This delocalization problem shows an inherent conceptual problem exists when reference densities need to be generated for anions. We shortly discuss this conceptual issue in Sec. III C and indicate if and how it is handled in other implementations. We present two effective solutions ourselves, and show how these improve the obtained results. In Sec. IIID the influence of the inclusion of core electrons in the reference densities is presented. As a last point, the atomic charges in some simple periodic systems are calculated, showing that the algorithm works correctly. Finally, in Sec. IV some conclusions are given.

\section{METHODS}

\section{A. Atomic and molecular calculations}

Hirshfeld-I calculations require EDDs as input. These can be obtained from electronic structure calculations using standard solid state or quantum chemical codes. In this work we have chosen to perform these calculations within the DFT framework using the projector augmented wave (PAW) approach for the core-valence interaction and the local density approximation (LDA) for the exchange-correlation functional as implemented in the VASP code. ${ }^{33,34}$ The kinetic energy cut-off is set at $500 \mathrm{eV}$ and the $k$-point set is reduced to the $\Gamma$-point for molecular and atomic calculations. For the bulk materials sufficiently converged $k$-point sets were used. To optimize the geometry of the molecules and periodic materials a conjugate gradient algorithm is applied. For molecular calculations only the atom positions are optimized, for bulk materials the cell parameters are optimized simultaneously. All molecules are placed in periodic cells of $20.0 \times 21.0 \times 20.5 \AA^{3}$, which provide a sufficiently large vacuum region between periodic copies of the molecules, to prevent interaction.

Hirshfeld-I data computed using the approach detailed in the present study, are compared to those obtained using more common molecular calculations of AIM properties. For the set of 168 neutral molecules previously studied by Bultinck et al., ${ }^{9,35}$ geometry optimization and Hirshfeld-I charge calculations are performed at the Local Spin Density Approximation ${ }^{36,37}$ level with the Slater exchange functional ${ }^{38}$ and the VWN5 correlation functional ${ }^{39}$ as implemented in Gaussian-03. ${ }^{40}$ Numerical integrations are carried out using Becke's integration grid with 170 angular points in the Lebedev-Laikov grid. ${ }^{41,42}$ The Hirshfeld-I charges are considered converged if the largest change in charge of any atom is below $0.0005 \mathrm{e}$.

\section{B. Hirshfeld methods}

The basic idea behind the Hirshfeld method, ${ }^{7}$ also known as the stockholder method, is that the AIM share the electron density in each point of space. This means that the AIM EDD becomes a weighted partition of the molecular EDD. Formally this can be written as:

$$
\begin{aligned}
\rho_{m o l}(\mathbf{r}) & =\sum_{A} \rho_{A}^{A I M}(\mathbf{r}) \\
\rho_{A}^{A I M}(\mathbf{r}) & =w_{A}^{H}(\mathbf{r}) \rho_{m o l}(\mathbf{r}) \quad \forall \mathbf{r} \\
\text { with } \sum_{A} w_{A}^{H}(\mathbf{r}) & \equiv 1,
\end{aligned}
$$

with $\rho_{\text {mol }}(\mathbf{r})$ and $\rho_{A}^{A I M}(\mathbf{r})$ the EDDs for the molecule and the AIM. All sums are taken over the entire set of AIM, and $w_{A}^{H}(\mathbf{r})$ is the Hirshfeld weight function for atom $A$. From these equations the Hirshfeld weight can be written as:

$$
w_{A}^{H}(\mathbf{r})=\frac{\rho_{A}^{A I M}(\mathbf{r})}{\sum_{B} \rho_{B}^{A I M}(\mathbf{r})} .
$$

Since the $\rho^{A I M}(\mathbf{r})$ are the EDDs sought, they cannot be used as input. The Hirshfeld method circumvents this problem by using spherically averaged reference state atomic EDDs $\rho_{X}^{\circ}(\mathbf{r})$. In the original paper by Hirshfeld the neutral atomic ground state is used as reference state. ${ }^{7}$ When summing these isolated atomic EDDs over all AIM, one gets the so-called 'promolecular' EDD instead of the actual molecular EDD:

$$
\rho_{\text {promol }}(\mathbf{r})=\sum_{B} \rho_{B}^{\circ}(\mathbf{r})
$$

It is then assumed that the difference between this promolecular EDD and the actual molecular EDD has only little influence on the Hirshfeld weight $w_{A}^{H}(\mathbf{r})$. As a result one can write the EDD of an AIM $A$ as:

$$
\rho_{A}^{A I M}(\mathbf{r})=\frac{\rho_{A}^{\circ}\left(n_{A}^{\circ}, \mathbf{r}\right)}{\rho_{\text {promol }}(\mathbf{r})} \rho_{m o l}(\mathbf{r}),
$$

where the population of the atom $A$ is given by $n_{A}^{\circ}$. In the original Hirshfeld approach, neutral atoms were used as reference. This, however, has been identified by several authors to be a major weakness of the method as changing the choice of the promolecular atom charges can have a highly significant effect on the resulting AIM $^{2,3,9,43,44}$ From eq.(4) it is easy to understand that the resulting $\rho_{A}^{A I M}(\mathbf{r})$ will tend to be as similar to $\rho_{A}^{\circ}\left(n_{A}^{\circ}, \mathbf{r}\right)$ as possible ${ }^{1,9,45}$, explaining why the Hirshfeld populations strongly depend on the choice of reference atomic EDDs. Fortunately, this problem can be resolved by using the iterative Hirshfeld-I scheme. ${ }^{8,9}$ For each iteration $i$, the obtained $\rho_{A}^{A I M}(\mathbf{r})$ are used to calculate the population 
$n_{A}^{i}$ of each atom $A$. The (spherically symmetric) EDD $\rho_{A}^{i}\left(n_{A}^{i}, \mathbf{r}\right)$ of a free atom $A$ with population $n_{A}^{i}$ is then used as atomic EDD in $w_{A}^{H}(\mathbf{r})$. For each iteration the new promolecular density $\rho_{\text {promol }}^{i}(\mathbf{r})$ is obtained by summing the density distributions $\rho_{A}^{i}\left(n_{A}^{i}, \mathbf{r}\right)$ for all atoms of the molecule. This setup is independent of the initial choice of atomic EDDs and the convergence of the iterative scheme is determined by the convergence of the populations of the AIM. ${ }^{46,47}$ Note that the first step in this scheme usually corresponds to the standard Hirshfeld method with $n_{X}^{\circ}=Z_{X}$. At this point it is important to note that the $\operatorname{EDDs} \rho_{A}^{A I M}(\mathbf{r})$ and $\rho_{A}^{i}\left(n_{A}^{i}, \mathbf{r}\right)$ will generally be different, despite having the same population. $\rho_{A}^{i}\left(n_{A}^{i}, \mathbf{r}\right)$ is constructed as a spherically symmetric EDD, whereas $\rho_{A}^{A I M}(\mathbf{r})$ is a weighted part of the molecular EDD. The resulting EDD will generally be not spherically symmetric but show protrusions along the directions bonds are formed.

The extension of the Hirshfeld and Hirshfeld-I methods from molecules to bulk and other periodic materials is quite trivial from the formal perspective. The main problem is located in the fact that a bulk system is considered to consist of an infinite number of "atoms in the system"(AIS). Calculating the atomic electron densities for all AIS can, for a periodic system, be reduced to only the atoms in a single unit cell since all periodic copies should yield the same results.

In addition to this, also the summation limits in Eqs. (2), (3) and (4) change. Where for molecules the sum over $B$ is a finite sum over all AIM, it becomes an infinite sum over all AIS. Because atomic EDDs drop exponentially, the density contribution to the "prosystem" $\operatorname{EDD} \rho_{\text {prosys }}(\mathbf{r})=\sum_{B}^{A I S} \rho_{B}^{\circ}\left(n_{B}^{\circ}, \mathbf{r}\right)$ of atoms at larger distances becomes negligible. This allows us to truncate the infinite sum to include only the atoms within a certain 'sphere of influence' (SoI), i.e. all atoms of which the contribution to the prosystem EDD is not negligible. Within the iterative Hirshfeld-I scheme we then get:

$$
w_{A}^{H, i}(\mathbf{r})=\frac{\rho_{A}^{i-1}\left(n_{A}^{i-1}, \mathbf{r}\right)}{\sum_{B}^{S o I} \rho_{B}^{i-1}\left(n_{B}^{i-1}, \mathbf{r}\right)} \quad \forall A \epsilon \text { unit cell }
$$

where

$$
\sum_{A}^{S o I} w_{A}^{H, i}(\mathbf{r}) \leq \sum_{A}^{A I S} w_{A}^{H, i}(\mathbf{r}) \equiv 1 \quad \forall \mathbf{r},
$$

with $i$ indicating the iteration step, and $\rho_{A}^{i}\left(n_{A}^{i}, \mathbf{r}\right)$ the atomic EDD for an atom $A$ with a population $n_{A}^{i}$ given by

$$
n_{A}^{i}=\int w_{A}^{H, i}(\mathbf{r}) \rho_{\text {system }}(\mathbf{r}) \mathrm{d} \mathbf{r}
$$

where $\rho_{\text {system }}(\mathbf{r})$ is the EDD of the periodic system.

\section{Spatial integration of the population}

In chemistry, due to the exponential decay of the electron density of atoms and molecules, and due to the sharp cusps present in EDDs at the atomic nuclei, atom centered grids are widely and successfully used. This makes them ideally suited for integrations such as given in Eq. (7). The multicenter integration scheme proposed by Becke splits up the full space integration into a set of overlapping atom centered spherical integrations. ${ }^{41}$ To solve the problem of double counting in the overlapping regions a weight $h_{A}(\mathbf{r})$ is given to each point in space for every atom $\mathrm{A}$ in the system, such that

$$
\sum_{A}^{A I S} h_{A}(\mathbf{r}) \equiv 1 \quad \forall \mathbf{r} .
$$

This weight function indicates how much a point 'belongs' to a certain atom $A$. The weight function can be binary, when the space is split up in Voronoi or Wigner Seitz cells, ${ }^{48,49}$ or smoothly varying, as is the case in the Becke scheme. ${ }^{41}$ As a result, an integrand $F(\mathbf{r})$ can be decomposed as $F(\mathbf{r})=\sum_{A}^{A I S} h_{A}(\mathbf{r}) F_{A}(\mathbf{r})$ and the full integration becomes

$$
I=\int F(\mathbf{r}) \mathrm{d} \mathbf{r}=\sum_{A}^{A I S} \int h_{A}(\mathbf{r}) F_{A}(\mathbf{r}) \mathrm{d} \mathbf{r},
$$

where the sum over all AIS is again an infinite sum. However, in numerical implementations for periodic systems, the exponential decay of the atomic EDD allows us to truncate both the infinite sum and integration region of Eq.(9), without significant loss of numerical accuracy. The sum can be reduced to contain only the atoms included in the SoI of atom $A$ (orange circles in Fig. 1), because only these atoms contribute significantly to the density in the integration region around atom $A$. In addition, the integration region for all atoms in the SoI can be reduced even further, without loss of accuracy, to only the region that overlaps with the spherical integration region of atom $A$ (blue shaded disc in Fig. 1).

\section{Grid stored electron densities and frozen core pseudo-potentials.}

In periodic systems, the use of $\mathrm{PBC}$ allows one to reduce the system size dramatically. For bulk materials this even allows simple systems, such as face-centered cubic $\mathrm{Cu}$ or $\mathrm{Ni}$, to be represented using single atom unit cells. A useful side effect of such reduced cells is that it is easily possible and relatively cheap to store the $\operatorname{EDD} \rho_{\text {system }}(\mathbf{r})$ on a three dimensional grid covering the unit cell, and thus fully describing the entire infinite system. The use of such precalculated electron density grids speeds up the Hirshfeld method significantly, since there is no more need to calculate the electron density at any given grid 


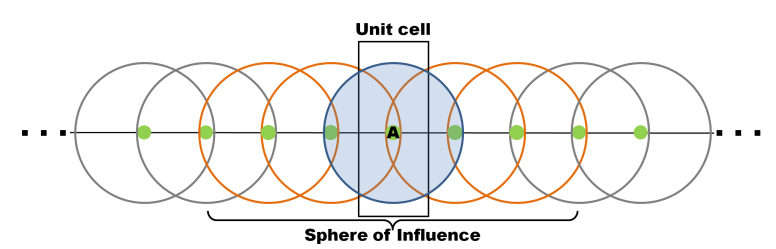

FIG. 1: (color online) Schematic representation of the integration scheme used for a linear periodic system of atoms (green discs). The black rectangle indicates the unit cell, and the circles indicate the boundaries of the spherical integration regions. The spherical integration regions for the atoms in the sphere of influence (SoI) of atom A are shown in orange, those outside are shown in grey. All integrations can be limited to the blue region (or sections thereof) around atom A (see text).

point starting from the wave function of the system. This also makes the implementation independent of the code used to generate the electron density. ${ }^{50}$ The drawback, however, is a slightly reduced accuracy. Since the electron density grid has a finite resolution, interpolation between the stored grid points is needed. This effect is discussed in the following section.

In chemistry, it is usually sufficient to consider only the valence electrons to describe the interactions between atoms. The core electrons are often considered inert as a result, and are kept frozen during calculations, greatly reducing the computational cost for heavy atoms. In the PAW approach an all electron frozen core contribution is used. This has two small but interesting side effects on the calculated radial charge distributions. Firstly, the integrated charge equals the number of valence electrons only, since the core electrons are not explicitly treated. Secondly, the resulting radial profile is not necessarily monotonically decreasing, showing a minimum or even negative values at the core of the atom (e.g. Fig. 4). The origin of this behavior lies in the practical implementation of the PAW pseudo-potentials. ${ }^{33,34}$ By using the same pseudo-potentials for generating the atomic EDDs and the EDD of the system under study, however, no errors are introduced since both systems contain the same frozen core contribution. Furthermore, it is known that for inorganic compounds, such as oxides, the oxygen atoms artificially absorb core electron density of (transition) metals. For this reason recent other Hirshfeld-I work in solids focused on valence densities only. ${ }^{31}$

Because the EDDs used in our implementation of the Hirshfeld-I method for periodic systems are valence only, and as a result the core electrons are not accounted for during the integration of the AIM densities, we implicitly add the core electrons exclusively to their respective atoms. On the one hand, this is fully in line with an atomic model where only the valence electrons are assumed to be involved in chemical interactions, such as bond formation. On the other hand, this can also be derived from the Hirshfeld weights defined in Eqn. (2). Consider the atomic EDD as the sum of the core and the valence densities, then Eqn. (2) can be written as:

$$
w_{A, c v}^{H}(\mathbf{r})=\frac{\rho_{A, \text { core }}^{A I M}(\mathbf{r})+\rho_{A, v a l}^{A I M}(\mathbf{r})}{\sum_{B}\left(\rho_{B, \text { core }}^{A I M}(\mathbf{r})+\rho_{B, v a l}^{A I M}(\mathbf{r})\right)} .
$$

For these atomic densities it is reasonable to assume that for a given atom $A$ the core electrons are contained within a certain core region $\mathbf{r}_{A \text {,core }}$. For any $\mathbf{r}>\mathbf{r}_{A \text {,core }}$ this means $\rho_{A, \text { core }}^{A I M}(\mathbf{r})$ is practically zero, and for $\mathbf{r}<\mathbf{r}_{A \text {,core }}$ it is reasonable to assume $\rho_{A, \text { core }}^{A I M}(\mathbf{r}) \gg \rho_{A, \text { val }}^{A I M}(\mathbf{r})>$ $\rho_{B, \text { val }}^{A I M}(\mathbf{r})$. As a result, Eqn. (10) can in both regions be reduced. If $\mathbf{r}<\mathbf{r}_{A, \text { core }}$ :

$$
\begin{aligned}
w_{A, c v}^{H}(\mathbf{r}) & =\frac{\rho_{A, \text { core }}^{A I M}(\mathbf{r})+\rho_{A, v a l}^{A I M}(\mathbf{r})}{\rho_{A, \text { core }}^{A I M}(\mathbf{r})+\sum_{B} \rho_{B, v a l}^{A I M}(\mathbf{r})} \\
& \cong \frac{\rho_{A, \text { core }}^{A I M}(\mathbf{r})}{\rho_{A, \text { core }}^{A I M}(\mathbf{r})}=1
\end{aligned}
$$

showing that within the core region the entire density is fully claimed by the respective atom. Outside the core region for $\mathbf{r}>\mathbf{r}_{A \text {, core }}$ one finds:

$$
w_{A, c v}^{H}(\mathbf{r})=\frac{\rho_{A, v a l}^{A I M}(\mathbf{r})}{\sum_{B} \rho_{B, v a l}^{A I M}(\mathbf{r})},
$$

which is the valence only version of the Hirshfeld weight as it was defined in Eqn. 2. In Sec. III D, we will test the validity of this assumption by comparing results of valence only EDDs with those of all electron EDDs.

\section{RESULTS AND DISCUSSION}

It is clear that there is a growing interest in codes that can provide atomic populations, however, only few true bulk systems have been investigated using a purely atom based Hirshfeld-I method ( $c f$. sec. I) ${ }^{12,16-31}$ For this reason most numerical tests in this work are performed on molecules, though we will investigate the behavior of periodic systems at the end of this section. As a first test system we have chosen the CO molecule. Its small size makes it easily suitable for quick test calculations, and its heteronuclear structure should result in a non-zero charge transfer, at least at equilibrium distance.

Before proceeding, the different grids used in our current setup of the Hirshfeld-I scheme for periodic systems are introduced. In this setup there are two 'types' of EDDs: that of the system and that of the free atoms/ions, which are indicated in the following as the subscripts 'sys' and 'atom', respectively. In this, 'system' refers to the object of which we want to obtain the atomic charges, and can thus refer to bulk materials, wires, molecules or even single ions. The free atoms/ions on the other hand refer to the single atoms which are used for the generation of the reference radial EDD $\rho_{X}^{i}$ of eqn.(5). For both types there are two kinds of $3 \mathrm{D}$ grids involved: 
1. Linear grids: Instead of using the analytical expression for the underlying wave function, we use the EDDs stored by VASP on a finite numerical grid. These grids span a single unit cell and use uniformly spaced grid points in direct coordinates. ${ }^{51}$ (cf. Sec.III A) In the remainder, the following notation is used:

- $\mathrm{V}_{\text {atom }}$ : The linear grid for the reference atom density distributions as obtained from the atomic calculations.

- $\mathrm{V}_{\text {sys }}$ : The linear grid for the (poly)atomic system under study

2. Spherical grids: These are atom centered grids which are not limited to a single unit cell. The spherical grids decompose into a radial and a shell grid. In our current setup, a logarithmic grid is used as radial grid, such that closer to the core the grid is sufficiently dense to describe this region accurately. The number of radial points was chosen to equal the numbers suggested by Becke. ${ }^{41}$ At each point in this radial grid, a shell is located on which grid points are distributed according to a Lebedev-Laikov grid. ${ }^{42}$ ( $c f$. Sec. IIIB) The total number of grid points $S$ equals the sum over all atoms of the number of radial points $\left(R_{A}\right)$ used for that atom times the number of points on each shell $(\sigma): S=\sum_{A} R_{A} \cdot \sigma$. In the remainder only $\sigma$ is varied to study the stability of the integrations. Two three-dimensional spherical grids are distinguished:

- $\mathrm{S}_{\text {atom }}$ : Total spherical grid used to generate the reference spherically averaged radial density distribution for the atoms.

- $\mathrm{S}_{\text {sys }}$ : The multi-center spherical grid for the system under study.

Note that the results of the Hirshfeld-I AIM analysis depend directly on $S_{\text {sys }}$ but also indirectly on $S_{a t o m}$ as this determines the quality of the isolated atomic EDDs.

\section{A. Electron density grids $\mathbf{V}_{\text {sys }}$ and $\mathbf{V}_{\text {atom }}$}

As was mentioned in the previous section, the use of precalculated grid-based EDDs introduces small inaccuracies due to the need for interpolation between the existing grid points. The charge of the $\mathrm{C}$ atom in a $\mathrm{CO}$ molecule as a function of the grid spacing used in the $\mathrm{V}_{\text {sys }}$ grid is shown in Fig. 2. The different curves are for different grid spacings used in the $\mathrm{V}_{\text {atom }}$ grids, from which the atomic radial EDD $\rho_{C}^{i}\left(n_{C}^{i}, \mathbf{r}\right)$ and $\rho_{O}^{i}\left(n_{O}^{i}, \mathbf{r}\right)$ are generated. It clearly shows the influence of both grids to be independent, since all curves have the same shape. Looking in detail at the exact numbers reveals that for both grids the same accuracy is obtained ( $c f$. Table I). This means that the same change in population of the

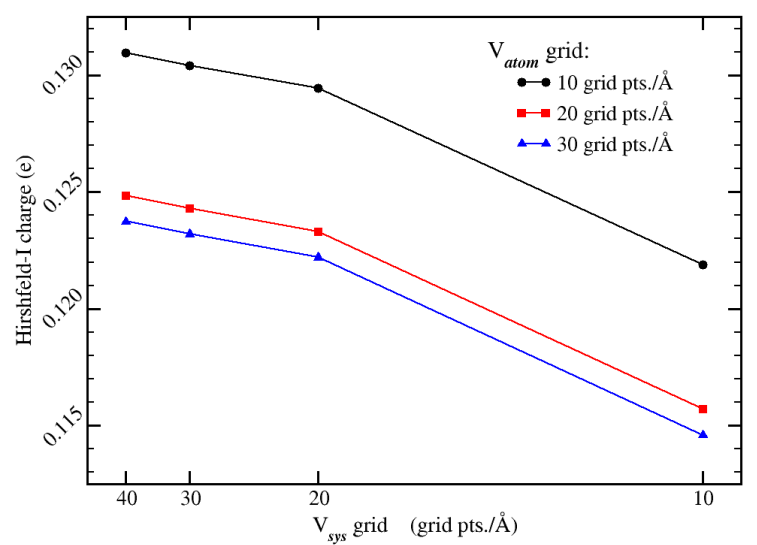

FIG. 2: The Hirshfeld-I charge for the $\mathrm{C}$ atom in a $\mathrm{CO}$ molecule as function of the $\mathrm{V}_{\text {sys }}$ grid resolution. The different curves show the results for the use of different resolutions in the $\mathrm{V}_{\text {atom }}$ grids, used for generating the atomic radial densities. In all molecular calculations we used spherical integration grids of 1202 grid points per shell.

TABLE I: Differences in the Hirshfeld-I populations shown in Fig. 2. The presented change in population is the difference in population going from $\mathrm{V}_{\text {sys }} / \mathrm{V}_{\text {atom }}$ grids with $I$ grid points per $\AA$, to grids with $J$ grid points per $\AA$. The respective grid changes are indicated as $\Delta_{J}^{I}$.

Top table: Differences along the curves shown in Fig. 2.

Bottom table: Differences between the curves shown in Fig. 2.

\begin{tabular}{|c|c|c|c|}
\hline \multirow[t]{2}{*}{$\mathrm{V}_{\text {sys }}$} & \multicolumn{3}{|c|}{$\mathrm{V}_{\text {atom }}($ grid points $/ \AA)$} \\
\hline & 10 & 20 & 30 \\
\hline$\Delta_{20}^{10}$ & 0.00738 & 0.00743 & 0.00744 \\
\hline$\Delta_{30}^{20}$ & 0.00126 & 0.00128 & 0.00128 \\
\hline$\Delta_{40}^{30}$ & 0.00049 & 0.00048 & 0.00049 \\
\hline $\begin{array}{c}\mathrm{V}_{\text {sys }} \\
(\text { grid points/A) }\end{array}$ & $\Delta_{20}^{10}$ & $\begin{array}{c}\text { Vatom } \\
\Delta_{30}^{20}\end{array}$ & \\
\hline 10 & -0.00617 & -0.00112 & \\
\hline 20 & -0.00612 & -0.00111 & \\
\hline 30 & -0.00610 & -0.00111 & \\
\hline 40 & -0.00611 & -0.00110 & \\
\hline
\end{tabular}

$\mathrm{C}$ atom in $\mathrm{CO}$ is observed when either the $\mathrm{V}_{\text {atom }}$ or the $\mathrm{V}_{\text {sys }}$ grid is changed in an identical way; i.e. the change in the population (in absolute value) of the $\mathrm{C}$ atom is comparable when going from the black to the red curve and when going from a point at 10 grid points per $\AA$ to a point at 20 grid points per $\AA$ on the same curve in Fig. 2. Figure 2 and Table I also show that quite a dense mesh is needed to obtain very accurate results. Though this is not a big problem for periodic systems with small unit cells, it could become problematic for molecules which require big unit cells to accommodate the vacuum required 

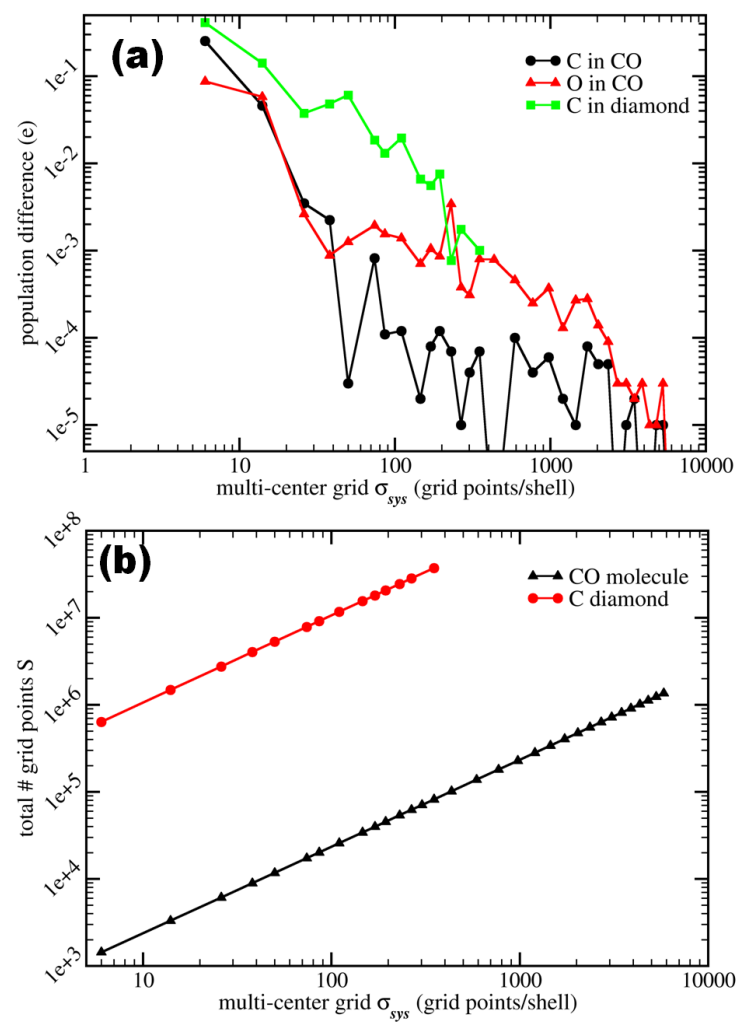

FIG. 3: (a) Convergence behavior of the population/charge as function of the number of grid points per spherical integration shell. For the $\mathrm{C}$ (black line) and $\mathrm{O}$ (red line) atom of the $\mathrm{CO}$ molecule the value shown is the absolute value of the difference between the calculated population and the calculated population using the most dense grid. In case of the $\mathrm{C}$ atom in the diamond system (green line) the charge should be zero, so the absolute value of the calculated charge is presented. (b) The total number of grid points as function of the number of Lebedev-Laikov grid points per spherical shell. ${ }^{42}$ The large number of grid points for the diamond system, is due to its large SoI (see text).

to prevent interaction between the periodic copies. The same is true for high accuracy $\mathrm{V}_{\text {atom }}$ grids, which are required for generating high accuracy atomic radial densities. Fortunately, these must only be calculated once, and the resulting high resolution radial densities can then be stored in a small size library containing only $\mathbf{r}$ dependent densities. In sec. III $\mathrm{C}$ the atomic radial EDDs are discussed in more detail.

\section{B. Spherical integration grids $\mathbf{S}_{\text {sys }}$ and $\mathbf{S}_{\text {atom }}$}

Because the populations and charges in the Hirshfeld(I) approach are obtained by integration of the EDD of the system, attention needs to be paid to the integration grid used. The current implementation uses a multi-centered grid, as was noted in Sec. II C. In this setup we used
Lebedev-Laikov grids on the spherical shells of the integration grids. ${ }^{42}$ Figure 3 a shows the influence of the number of grid points per spherical shell on the accuracy; note the log-log scale used. In general, a denser grid results in a more accurate value for the population. However, different atoms in the same system, and the same calculation can show different convergence, as is shown by the curves of the $\mathrm{C}$ and $\mathrm{O}$ atoms of a $\mathrm{CO}$ molecule. To have the population of the system atoms converged to within 0.001 electron a few hundred grid points per shell are required. For a molecular calculation the multi-center grid, $\mathrm{S}_{\text {sys }}$, only requires a few tens of thousands of grid points, as is seen in Fig. 3b. However, a Hirshfeld-I calculation for a bulk material such as diamond, which also has only two atoms in its unit cell, requires a multi-center grid $\mathrm{S}_{\text {sys }}$ with several tens of millions of grid points. This difference by a factor thousand originates from the fact that the SoI for the diamond unit cell contains a few thousand atoms, which all contribute to the total number of grid points that need to be evaluated. This makes it very important to reduce the SoI as much as possible size without significant loss of accuracy.

\section{Atomic radial EDDs}

The atomic EDDs are calculated in a periodic cell under $\mathrm{PBC}$, and stored on 3D electron density grids $\mathrm{V}_{\text {atom }}$ ( $c f$. Sec. III A). The atomic radial EDDs are obtained from these through spherical averaging of the density distributions. Spherical averaging is done using the $\mathrm{S}_{\text {atom }}$ grid, with spherical shells containing LebedevLaikov grids of 5810 grid points. ${ }^{42}$ The resulting distributions for different $\mathrm{C}$ ions are shown in Fig. 4a. The populations obtained by spherical integration over these distributions shows that the correct populations are obtained for the neutral and the positively charged ions. In contrast, the negatively charged ions show a population which is too small. Moreover, the curves in Fig. 4a increase again for longer ranges. At first glance, one might attribute this behavior entirely to the overlapping tails of periodic copies in neighboring cells. However, a rough extrapolation of the decreasing part of the curve (multiplied with the number of neighboring cells) shows a much lower electron density at a distance of $10 \AA$ than is currently the case. The actual origin of the increase lies in the fact that the plane wave approach used for the atom calculations can only bind a limited amount of extra electrons to a given atom. This amount varies from atom to atom. As a result, it tries to place the excess electrons as far from the atom as possible. Due to strong delocalization inherent to plane waves these electrons are spread out over the vacuum between the atoms, with the highest electron density at the center of the unit cell; i.e. as far from the atoms as possible.

It is interesting to note that this artifact is purely due to the use of plane waves, which try to smear out the unbound electrons over the entire (empty) space. If one 

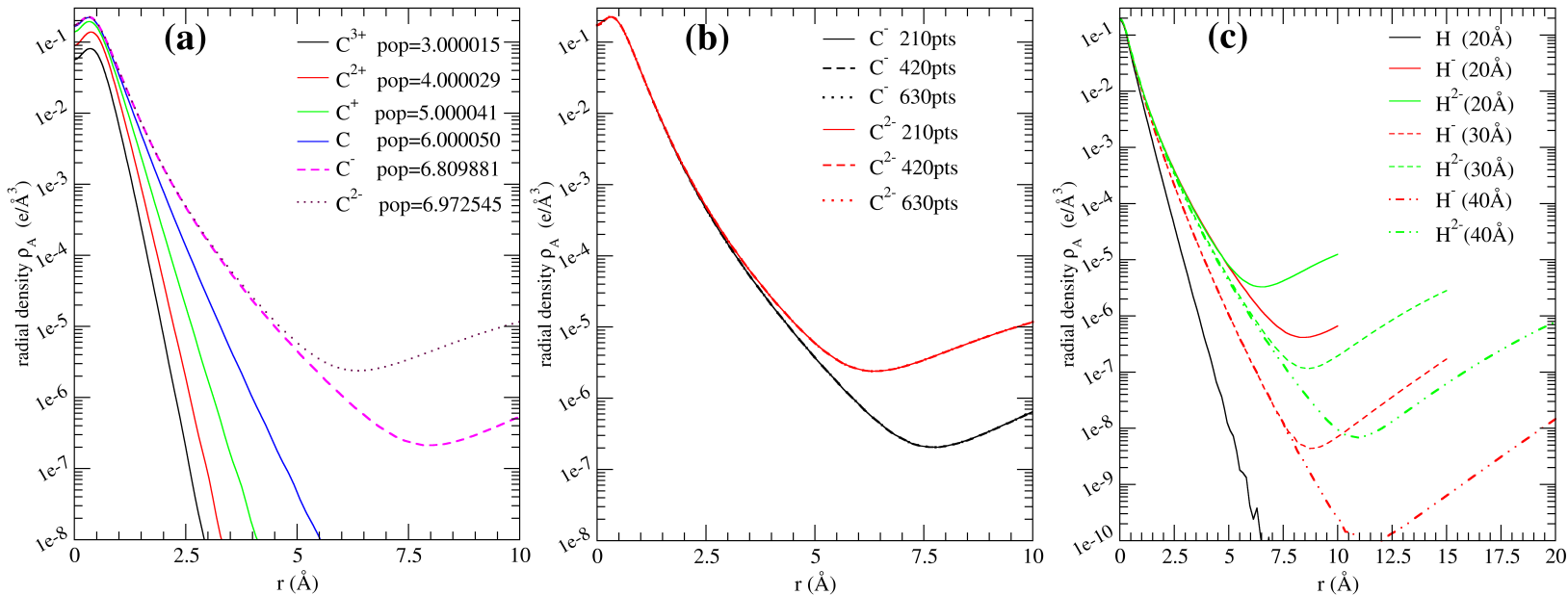

FIG. 4: Atomic radial electron densities $\rho_{A}(r)$ calculated from grid stored atomic charge distributions. (a) Radial electron densities for different carbon ions obtained from charge distributions in a cubic periodic unit cell of $20.5 \times 20.5 \times 20.5 \AA^{3}$. The populations (pop) given of each ion are calculated by spherical integration of this radial distribution. (b) Radial charge distributions for two negative carbon ions obtained from the same size periodic unit cell as (a), but with different grid spacings:

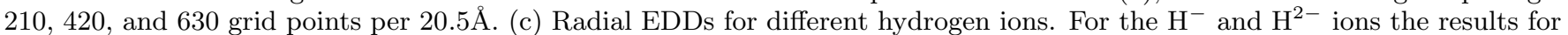
different unit cell sizes are compared: $20 \times 20 \times 20 \AA^{3}, 30 \times 30 \times 30 \AA^{3}$, and $40 \times 40 \times 40 \AA^{3}$.

uses a (finite) Gaussian basis set instead, the additional electrons are 'bound' by definition through the basis set used, even if these electrons should not be bound to the atom anymore. In addition there looms also a conceptual dilemma: should one use such 'artifacts' as reference states or should one avoid them and restrict oneself only to reference systems that can exist. For example, take the $\mathrm{O}^{2-}$ anion. In the gas phase, the second electron is unbound by over $8 \mathrm{eV}$ and one may suggest to use the $\mathrm{O}^{-}$electron density instead. However, the computational repercussion of this would be that the HirshfeldI method would become unstable for systems where a higher charge is found than is available as ground state free anion. This behavior and conceptual dilemma is not unique to Hirshfeld-I. Other approaches using external reference atomic densities suffer it too, since it is inherent to the generation of external atomic reference densities (e.g. DDEC-code, ${ }^{12}$ although this is not investigated by those authors). Some other approaches, like the iterative stockholder approach (ISA) of Lillestolen and Wheatley, appear to sidestep this dilemma by generating reference atomic densities as part of the iterative process. ${ }^{10,11}$ The drawback, in comparison to Hirshfeld-I, of such an approach is that the transferability of the reference atoms is lost. Also, the physical meaning of the reference atom in ISA becomes unclear. On the other hand, the ISA approach shows that the reference atomic densities are to be considered first and foremost 'tools'. This should alleviate the pressure on our conceptual conscience a little. Returning to the atomic reference densities used in
Hirshfeld-I, it is possible to define them as the density of free ions in the gas phase. This definition is then softened such that also useful densities can be obtained beyond the range of ground state ions in the gas phase. This can be done in several ways: using meta-stable anion densities, binding the additional electrons through the basis set (Gaussian approach), or keeping the shape-function of the first anion and scaling it in an appropriate way such that the required number of electrons is found on integration. These last two methods correspond to the approaches presented at the beginning of this section.

From the modeling point of view one might prefer this latter type of pragmatism over the former, since we are interested in the EDD of the electrons for 'free ions', irrespective of their bound or unbound nature. Later in this section we will show how this delocalization problem can be solved in a simple way.

Fig. 4b shows that this artifact is independent of the resolution used for the $\mathrm{V}_{\text {atom }}$ grid, as the different curves nicely overlap. Fig. 4c shows the influence of the periodic cell size on this artefact. In case of the presented hydrogen ions, it shows that using a cubic periodic cell with a side of $20 \AA$, the curves for $\mathrm{H}^{-}$and $\mathrm{H}^{2-}$ coincide in the short range region. Moreover, they don't show the expected exponential decay, and increase sharply at longer range. Using a larger unit cell appears to solve these problems: firstly, the radial distributions for the two anions become distinguishable, and show the expected exponential decay. Secondly, the point where the excess electrons start to interfere noticeably is pushed back to 

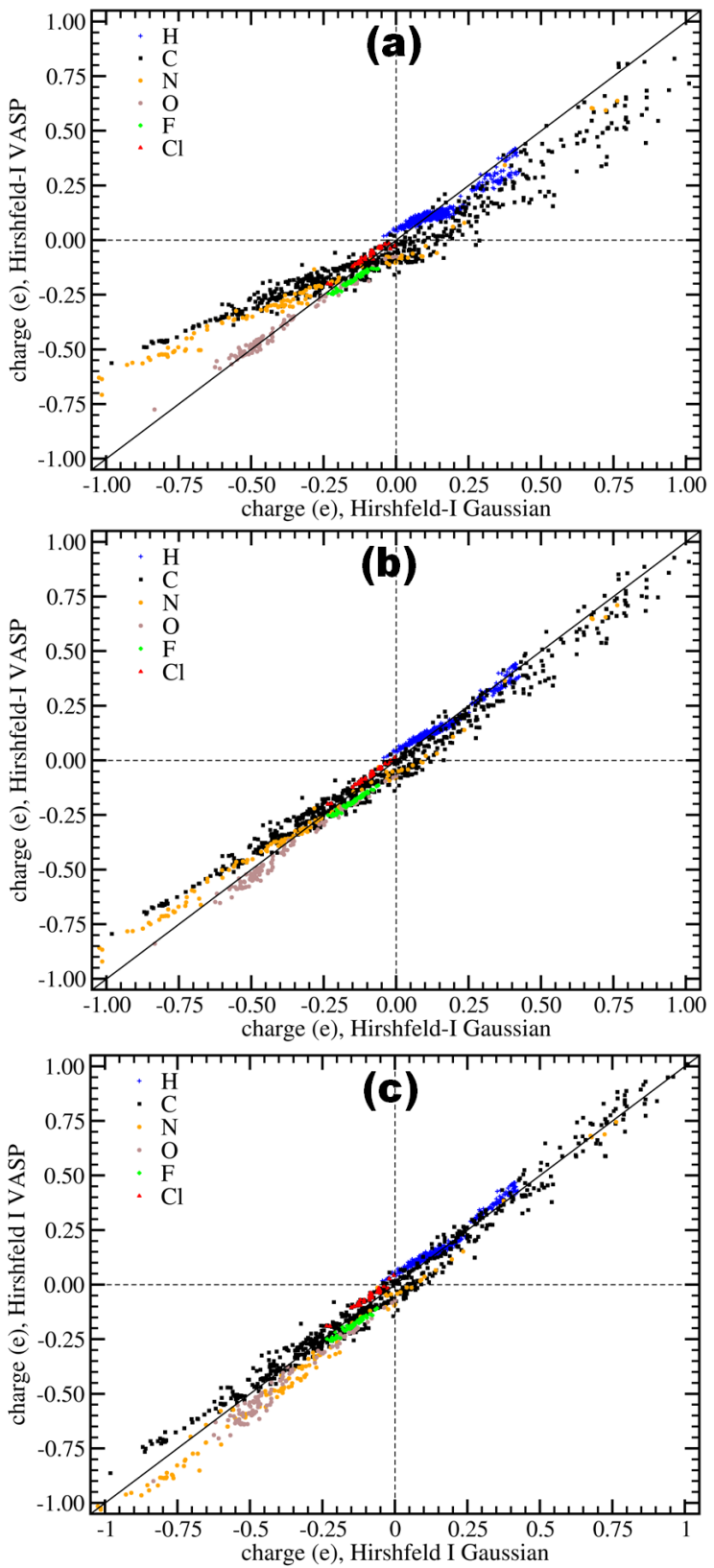

FIG. 5: Correlation plots of the charges obtained using the periodic Hirshfeld-I implementation and the standard all electron molecular implementation: (a) using the atomic radial density distributions of set R2 with the corrected tails, (b) using the R3 set with the corrected tails and normalized distributions assuming correct shape functions, and (c) using the R4 set with corrected tails and anion distributions normalized assuming piecewise linear interpolation of fractional densities (see text). In each case the VASP optimized geometry was used for the VASP based Hirshfeld-I calculations, while Gaussian optimized geometries were used for the reference calculations.

a larger distance. At this point it is interesting to note that for the DDEC-code this problem is not mentioned, although the references densities in the $\mathrm{c} 1$ method appear to be obtained in the same fashion as presented here. ${ }^{12}$ Furthermore, from Fig. 4 it is clear that large unit cells are required for calculating correct radial reference densities for anions. For both $\mathrm{C}$ and $\mathrm{H}$ anions it is shown that a significant charge density is still present at a distance of $7-10 \AA$ from the nucleus making unit cells of $20 \times 20 \times 20$ $\AA^{3}$ necessary to prevent additional overlap errors.

This type of behavior is seen for all atom types investigated, positive up to neutral ions give the expected radial distributions, while the negative ions seem hampered by the fact that only a fraction of the additional electrons can be attached to the atom. Fluorine and chlorine are in this respect exceptional since for these atoms also the $\mathrm{F}^{-}$and $\mathrm{Cl}^{-}$ions give good distributions and populations. This could be considered a result of the high electron affinity of these elements.

We find that for most negatively charged ions the populations are too small. ${ }^{52}$ As a result, the calculated Hirshfeld weight $w_{A}^{H}(\mathbf{r})$ for a negatively charged AIM $\mathrm{A}$ is underestimated. The easiest way to compensate for this discrepancy is by scaling these specific distributions such that the correct population is found after integration. This way the shape function of the curve is maintained, ${ }^{53,54}$ but the resulting weights $w_{A}^{H}(\mathbf{r})$ increase. Another possible approach is based on the assumption that the fractional density is piecewise linear between integer charges. ${ }^{9,55,56}$ This gives rise to an alternative way of normalizing the anion radial densities to present the correct integer charges. To investigate the effects of such a normalization and the erroneous tails shown in Fig. 4, we compare the results of four types of atomic radial density distributions. To that end, the reference set of molecules previously used in Hirshfeld-I studies by Bultinck et al..$^{9,35}$ is used. This set consists of 168 neutral molecules containing only $\mathrm{H}, \mathrm{C}, \mathrm{N}, \mathrm{O}, \mathrm{F}$, and $\mathrm{Cl}$.

The first set of atomic radial EDDs, called R1, contains the density distributions as shown in $4 \mathrm{a}$, where the radial distribution is obtained from a periodic cell of $20 \times 20 \times 20 \AA^{3}$.

For the second set, which is referred to as R2, we have combined these results with the results from a periodic cell of $40 \times 40 \times 40 \AA^{3}$ but with a lower grid resolution. In this case, the central part of the radial distribution is taken from the $20 \times 20 \times 20 \AA^{3}$ unit cell with the high resolution grid and connected to the tail part obtained from the $40 \times 40 \times 40 \AA^{3}$ unit cell. As a result, the high accuracy for the center part of the distribution is maintained, and the tail is corrected through the removal of the delocalized electron contribution. Note that for these distributions, the curves are limited to a distance of $10 \AA$ from the core, i.e. the same maximum radius as is available from the $20 \times 20 \times 20 \AA^{3}$ periodic cells. Because the excess tail electrons are not included anymore, the spherically integrated populations of the negative ions are slightly smaller than they are for the R1 set.

For the third set, R3, the same procedure as for R2 is used, but this time the curves are normalized for the 
negative ions such that the correct population is given on spherical integration of these radial EDDs while retaining the original shape function.

The fourth set, R4, is obtained using the same procedure as for R2, fixing the tail part, and like R3 the resulting curves are then normalized for the anions. In this case, however, the starting assumption for this last step is that the fractional density is given by:

$\rho(n+x, \mathbf{r})=(1-x) \rho(n, \mathbf{r})+x \rho(n+1, \mathbf{r}) \quad n \in \mathbb{N}, x \in[0 . .1]$,

where $n+x$ gives the fractional population. In the present situation, $\rho(n, \mathbf{r})$ represents the density distribution of the neutral atom, and $\rho(n+x, \mathbf{r})$ the obtained density distribution for the anion. Note that for the $\rho(n+x, \mathbf{r})$ distribution the spurious tail has already been fixed at this point, resulting in the expected exponentially decreasing tail. Rewriting Eqn. 14 gives:

$\left.\left.\rho(n+1, \mathbf{r})=\frac{\rho(n+x, \mathbf{r})-(1-x) \rho(n, \mathbf{r})}{x} \quad n \in \mathbb{N}, x \epsilon\right] 0 . .1\right]$.

The resulting $\rho(n+1, \mathbf{r})$ yields the full anion charge of $n+1$ upon spherical integration.

To test the accuracy of the results obtained in our periodic implementation using these four different atomic radial density distribution sets, we compare the results for a large benchmark set of molecules with those obtained by a Hirshfeld-I implementation based on a molecular program ( $c f$. Sec. II A).

First, two sets of calculations are performed to check the influence of the geometry on the results. The first set used the optimized geometries obtained from Gaussian calculations, and the second set used optimized geometries obtained through VASP calculations. For both sets the R1 atomic radial distributions are used. Table II shows that the resulting correlations are nearly identical, despite the small differences in geometries for the two sets. Although the correlation coefficients $r$ can be considered reasonable, the spread on the $\mathrm{C}$ and $\mathrm{N}$ data points is quite large. Furthermore, the slope of the linear fit of the $\mathrm{C}$ and $\mathrm{N}$ data is much too big. In both cases the intercepts are acceptably small.

Looking at the effect of fixing the tail of the atomic distributions, through the use of the $\mathrm{R} 2$ set of atomic radial density distributions, we see a slight increase in the slope for the $\mathrm{C}$ and $\mathrm{N}$ data, but in general the obtained values for the correlation, intercept and slope remain comparable to the previous sets of calculations. For the results obtained with the $\mathrm{R} 2$ radial distributions, Table II also shows the standard deviation of the difference between our calculated results and those of the reference data. The values of these standard deviations are similar for the first two sets, which are therefore not shown. Table II shows the deviation for the $\mathrm{C}$ and $\mathrm{N}$ data sets to be one order of magnitude larger than for the other elements. It is unclear to the authors why specifically these two elements show such a bad behavior. Looking at the underestimation of the atomic population for the mono-

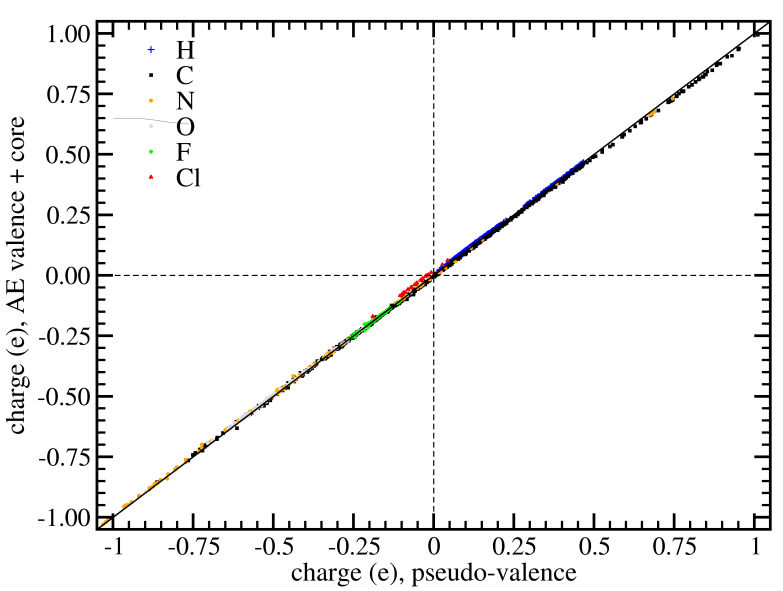

FIG. 6: Comparison of Hirshfeld-I charges for a set of 168 molecules using pseudo valence densities (method R4) and all electron (AE) valence+core densities.

valent anions we find an error of roughly $0.5 \mathrm{e}$ for $\mathrm{H}$ and $\mathrm{N}$, and $0.3 \mathrm{e}$ for $\mathrm{C}$ and $\mathrm{O}$. On the other hand for $\mathrm{F}$ and $\mathrm{Cl}$ an underestimation of 0.1 and $0.0 \mathrm{e}$, respectively, is found. The only aspect in which $\mathrm{C}$ and $\mathrm{N}$ differ from the other elements is that both can show large positive and negative charges.

The correlation results of the $\mathrm{R} 3$ atomic radial density distributions show a clear improvement over the previous results. For each data set the slope is closer to unity, though the intercepts remain as before. Especially the $\mathrm{C}$ and $\mathrm{N}$ results show a large improvement. Their standard deviation gets halved which is clearly visible in the correlation plots in Fig. 5a and b. This immediately shows that the simple scaling used for fixing the underestimated population due to the delocalization problem does not introduce large artifacts. In contrast, it actually suggests that the shape function of the radial EDD of the negatively charged ions contains roughly the same information as the Gaussian all electron EDD.

The results obtained with the R4 set show even better results. The correlation plot in Fig. 5c shows improved slopes for the $\mathrm{C}$ and $\mathrm{N}$ data. The slopes presented in Table II are closer to unity for nearly all elements, while small values for the intercepts are retained. The correlation and the standard deviation, however, have stayed roughly the same in comparison to the results of the R3 set. This shows that the R4 set can be used as a good approximation for the gaussian all electron EDDs.

\section{Inclusion of core electrons}

From Eqs. (10)-(12) it was deduced that the atomic core density can be exclusively added to the atom it originates from. Furthermore, it was show that only the valence electrons are shared between different atoms, giving rise to the obtained atomic charges, in line with chemical intuition. To test the validity of this deduction, we 
TABLE II: The fitting and correlation results for the different sets of radial EDDs used in Hirshfeld-I calculations for a set of 168 molecules. The molecular geometries are either optimized with Gaussian or VASP. The radial distributions R1 are the default distributions obtained from VASP atomic density distributions. The radial distributions R2 contain a fix for the tail of the distribution, and the radial distributions R3 contain a fix for the tail of the distributions and in addition the resulting distributions are normalized to give the correct number of electrons (see text). The radial distributions R4 contain the same tail fix as R2 and R3, but the normalization makes use of the assumption that fractional densities show a piecewise linear behavior (see text). $a$ and $b$ are the slope and the intercept of the linear fit. $r$ is the correlation with Gaussian molecular results, and $\sigma$ gives the standard deviation.

\begin{tabular}{|c|c|c|c|c|c|c|c|c|c|c|}
\hline & \multirow{2}{*}{\multicolumn{3}{|c|}{$\begin{array}{c}\text { Gaussian geometry } \\
\text { R1 }\end{array}$}} & \multicolumn{7}{|c|}{ VASP geometry } \\
\hline & & & & & R1 & & & & & \\
\hline & $a$ & $b$ & $r$ & $a$ & $b$ & $r$ & $a$ & $b$ & $r$ & $\sigma$ \\
\hline$\overline{\mathrm{H}}$ & 1.187 & -0.014 & 0.940 & 1.208 & -0.019 & 0.944 & 1.185 & -0.008 & 0.933 & 0.026 \\
\hline $\mathrm{C}$ & 1.353 & 0.020 & 0.966 & 1.344 & 0.026 & 0.970 & 1.439 & 0.020 & 0.963 & 0.181 \\
\hline $\mathrm{N}$ & 1.454 & 0.026 & 0.978 & 1.455 & 0.018 & 0.979 & 1.483 & 0.025 & 0.975 & 0.154 \\
\hline $\mathrm{O}$ & 1.131 & 0.066 & 0.992 & 1.123 & 0.057 & 0.991 & 1.152 & 0.064 & 0.992 & 0.031 \\
\hline $\mathrm{F}$ & 1.110 & 0.064 & 0.982 & 1.150 & 0.072 & 0.977 & 1.197 & 0.070 & 0.981 & 0.011 \\
\hline $\mathrm{Cl}$ & 1.117 & -0.009 & 0.979 & 1.124 & -0.018 & 0.980 & 1.110 & -0.013 & 0.966 & 0.015 \\
\hline
\end{tabular}

\begin{tabular}{|c|c|c|c|c|c|c|c|c|}
\hline & \multicolumn{8}{|c|}{ VASP geometry } \\
\hline & & & & & & & & \\
\hline & $a$ & $b$ & $r$ & $\sigma$ & $a$ & $b$ & $r$ & $\sigma$ \\
\hline $\mathrm{H}$ & 1.109 & -0.021 & 0.979 & 0.016 & 0.997 & -0.016 & 0.980 & 0.014 \\
\hline $\mathrm{C}$ & 1.131 & 0.014 & 0.989 & 0.086 & 1.027 & 0.003 & 0.991 & 0.057 \\
\hline $\mathrm{N}$ & 1.161 & 0.033 & 0.995 & 0.072 & 0.967 & 0.052 & 0.995 & 0.031 \\
\hline $\mathrm{O}$ & 1.025 & 0.048 & 0.993 & 0.019 & 0.947 & 0.042 & 0.984 & 0.027 \\
\hline $\mathrm{F}$ & 1.162 & 0.071 & 0.987 & 0.009 & 1.131 & 0.064 & 0.974 & 0.013 \\
\hline $\mathrm{Cl}$ & 1.072 & -0.018 & 0.993 & 0.007 & 1.024 & -0.032 & 0.979 & 0.011 \\
\hline
\end{tabular}

have calculated the atomic charges for the set of benchmark molecules using the total all electron densities, and compared the results to those obtained with the R4 reference densities. Reference all electron core and valence densities are obtained in a similar way as before, and are summed to provide full all electron reference densities. ${ }^{57}$ The same is done for the system EDDs, i.e. all electron core and valence densities are added to result in full all electron EDDs. As can be seen in Fig. 6, the obtained Hirshfeld-I charges for the valence calculations of the previous section, show excellent agreement with the full all electron results. The differences in the obtained atomic charges are generally below $0.01 e$, and always smaller than the differences due to the use of a too coarse $\mathrm{V}_{\text {sys }}$ grids. This clearly shows the assumption based on Eqs. (10)-(12) is a valid one to make.

\section{E. Periodic systems}

In this final section some actual periodic systems are considered. The choice of the example systems is such that they can be used to verify that the obtained results are reasonable. As such these systems are in essence quite trivial. If they were not, then any results presented would be meaningless numbers, unless reference values are available in literature, of which there are at the time of writing very few. One might be tempted to use indirect ways of trying to verify the results (e.g. ESP fitting), but this generally complicates matters and

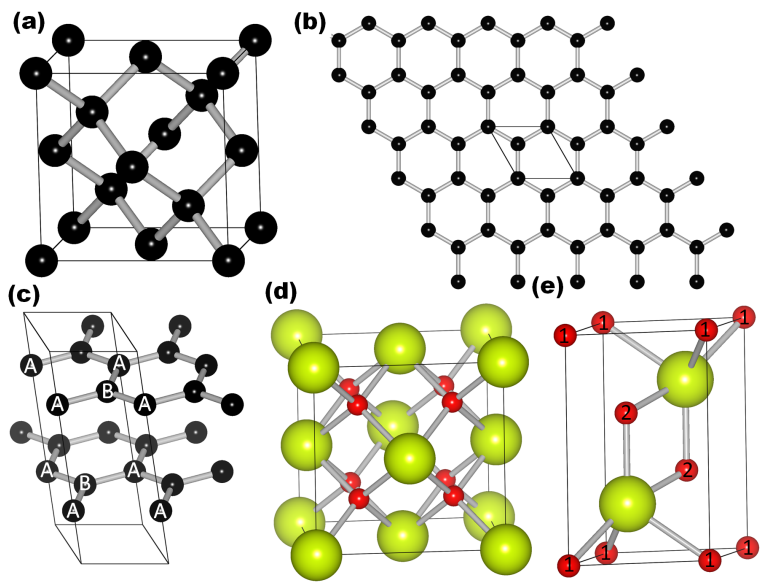

FIG. 7: Ball-and-stick representations of (a) the cubic diamond super cell, (b) the graphene sheet (unit cell indicated with black parallelogram), (c) graphite (unit cell indicated) in black, (d) the cubic $\mathrm{CeO}_{2}$ super cell, and (e) the $\mathrm{Ce}_{2} \mathrm{O}_{3}$ unit cell. The black, red and yellow spheres indicate the positions of the carbon, oxygen and cerium atoms,respectively. The inequivalent $\mathrm{C}$ atoms in the graphite structure are indicated as $A$ and $B$. The $B$ atoms are always located at the center of the hexagons of the neighboring sheets. The inequivalent $\mathrm{O}$ positions in $\mathrm{Ce}_{2} \mathrm{O}_{3}$ are indicated as 1 and 2 .

The ball-and-stick representations are generated using the VESTA visualization tool. ${ }^{58}$

obfuscates the validity of the results. In addition, small 
simple systems also keep the obtained results clear. For these reasons we have chosen the systems presented below. Their structures are presented in Fig. 7: the systems of choice are diamond, graphene, graphite, $\mathrm{CeO}_{2}$, and $\mathrm{Ce}_{2} \mathrm{O}_{3}$. The diamond and graphene systems, have the property that all $\mathrm{C}$ atoms are equivalent, which should result in zero charges on all atoms. Graphite is quite similar to graphene, however, two inequivalent $\mathrm{C}$ positions are present ( $c f$. Fig. 7c). The ceria systems on the other hand are chosen for the presence of Ce atoms with different valency; tetravalent $\mathrm{Ce}$ in $\mathrm{CeO}_{2}$ and trivalent $\mathrm{Ce}$ in $\mathrm{Ce}_{2} \mathrm{O}_{3}$. Note that for both systems the same Ce pseudo-potential is used. Furthermore, in $\mathrm{CeO}_{2}$, the $\mathrm{O}$ ions are all equivalent, while in $\mathrm{Ce}_{2} \mathrm{O}_{3}$ the $\mathrm{Ce}$ ions are equivalent but the $\mathrm{O}$ ions are not, only two of them are equivalent. Furthermore, for $\mathrm{Ce}_{2} \mathrm{O}_{3}$ we consider both the ferromagnetic (FM) and anti-ferromagnetic (AF) configurations, allowing us to check how strongly different spin-configurations influence the results in this system.

For all these systems we use the radial atomic EDDs of the R3 set, with the $\mathrm{S}_{\text {atom }}$ spherical integration grids containing 5810 grid points per shell. Table III shows the $k$-point sets used for the periodic systems, the number of atoms per unit cell and the number of atoms included in the SoI. The grid point separation for the $\mathrm{V}_{\text {sys }}$ grid for each of these systems was set to $\leq 0.01 \AA$. The Hirshfeld(-I) populations are calculated using spherical integration grids $\mathrm{S}_{\text {sys }}$ with 1202 Lebedev-Laikov grid points per shell.

The large number of atoms in the SoI with regard to the small unit cell sizes might make one wonder if this method can efficiently be used for larger (more interesting) systems. Due to the very nature of the SoI it are the small unit cells which have the highest cost per system cell atom. As the system cell grows, the relative weight of the SoI decreases and as a result it becomes relatively cheap to handle large supercells. This is clearly demonstrated in Fig. 8 where the scaling of the $\mathrm{CPU}$ time and the SoI is presented for a set of diamond system cells of varying size. The smallest cell is the unit cell containing 2 atoms, while the largest cell is a super cell of 128 atoms. Because our implementation does not make use of symmetry, a system containing 100 equivalent atoms is treated the same way as one containing 100 inequivalent atoms. This allows us to use the diamond system to test the scaling of the implementation. In addition, since all supercells present 'the same system', an equal number of Hirshfeld iteration are performed for all supercells to obtain convergence, making them ideally suited to check the scaling behavior. As can be seen in Fig. 8, going from the unit cell to the largest super cell the SoI roughly doubles, while the system cell has become $64 \times$ larger. On the other hand, the CPU time required for the Hirshfeld-I calculation is only increased roughly tenfold, again showing the beneficial trend for larger systems. From this it may be clear that this method is well suited for large systems, and we

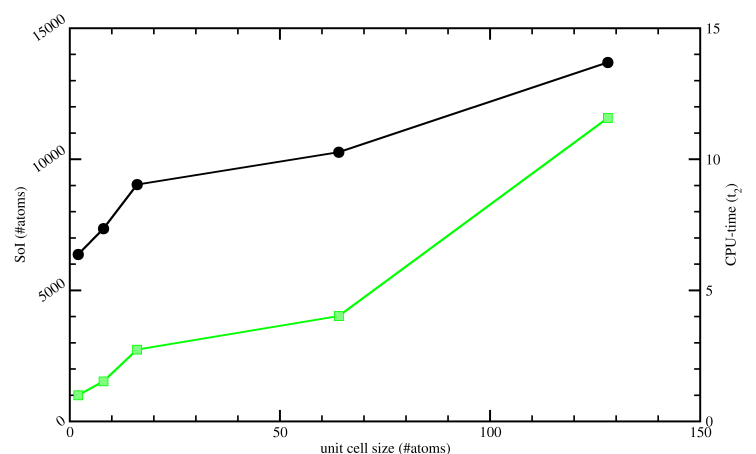

FIG. 8: Scaling behavior of the implemented Hirshfeld-I method. Black discs show the SoI in function of the system cell size (left axis), while the green squares show the required CPU time normalized with regard to the unit cell calculation (right axis).

TABLE III: $k$-point sets and the number of atoms in the unit cell and SoI for the periodic systems under investigation. In addition, also the total number of grid points used for the spherical integration grid are given.

\begin{tabular}{l|rrrccc}
\hline \hline & $k$-point set & $\begin{array}{c}\text { atoms per } \\
\text { unit cell }\end{array}$ & $\begin{array}{c}\text { atoms in } \\
\text { the SoI }\end{array}$ & $\begin{array}{c}\text { grid points } \\
\left(\times 10^{6}\right)\end{array}$ \\
\hline diamond & \multicolumn{2}{|c}{$21 \times 21 \times$} & 21 & 2 & 6374 & 128 \\
graphene & $21 \times 21 \times$ & 1 & 2 & 1276 & 22 \\
graphite & $21 \times 21 \times$ & 11 & 4 & 4618 & 104 \\
$\mathrm{CeO}_{2}$ & $8 \times$ & $8 \times$ & 8 & 3 & 3063 & 69 \\
$\mathrm{Ce}_{2} \mathrm{O}_{3} \mathrm{FM}$ & $10 \times 10 \times$ & 5 & 5 & 3009 & 77 \\
$\mathrm{Ce}_{2} \mathrm{O}_{3} \mathrm{AF}$ & $10 \times 10 \times$ & 5 & 5 & 3025 & 78 \\
\hline \hline
\end{tabular}

expect it could easily handle systems containing a few thousand atoms in the system cell. (Although this might not be the case for the solid state or quantum chemistry code used to provide the required EDDs.)

However, to investigate the obtained results we opted for small systems. The resulting Hirshfeld and HirshfeldI charges for the inequivalent atoms are shown in Table IV. It clearly shows the Hirshfeld values are closer to zero (i.e. the charge at which the atoms are initialized) than the Hirshfeld-I ones. This is the expected behavior and its origin was discussed earlier by Ayers ${ }^{1}$ and Bultinck et al. ${ }^{9}$ The diamond and graphene charges are (nearly) zero as one would expect based on symmetry arguments. This shows there are no significant artifacts which introduce spurious charges due to the PBC. The results for graphite are somewhat remarkable. Table IV shows there is a small charge transfer going from the A to the B sites. This could be understood as a consequence of the very weak bonding between the A sites in different sheets. For each $\mathrm{C}$ atom, three electrons are placed in hybridized $s p^{2}$ orbitals, where the fourth electron delocalizes in distributed $\pi$ bonds. For the A site 
TABLE IV: Hirshfeld and Hirshfeld-I charges calculated using LDA generated EDDs. The geometries of the periodic systems are shown in Fig. 7 where the labels for the inequivalent atoms are given.

\begin{tabular}{ll|rr}
\hline \hline & & $\begin{array}{c}\text { Hirshfeld } \\
(\mathrm{e})\end{array}$ & \multicolumn{1}{c}{$\begin{array}{c}\text { Hirshfeld-I } \\
(\mathrm{e})\end{array}$} \\
\hline diamond & $\mathrm{C}$ & -0.00007 & -0.00007 \\
graphene & $\mathrm{C}$ & 0.00000 & 0.00000 \\
graphite & $\mathrm{C}_{A}$ & 0.00113 & 0.00705 \\
& $\mathrm{C}_{B}$ & -0.00115 & -0.00707 \\
$\mathrm{CeO}_{2}$ & $\mathrm{Ce}$ & 0.59463 & 2.79393 \\
& $\mathrm{O}$ & -0.30091 & -1.40056 \\
& $\mathrm{Ce}$ & 0.49081 & 2.32119 \\
$\mathrm{Ce}_{2} \mathrm{O}_{3} \mathrm{FM}$ & $\mathrm{O}_{1}$ & -0.31318 & -1.61119 \\
& $\mathrm{O}_{2}$ & -0.33576 & -1.51663 \\
& $\mathrm{Ce}$ & 0.48575 & 2.32755 \\
$\mathrm{Ce}_{2} \mathrm{O}_{3} \mathrm{AF}$ & $\mathrm{O}_{1}$ & -0.30825 & -1.63062 \\
& $\mathrm{O}_{2}$ & -0.33317 & -1.51378 \\
\hline \hline
\end{tabular}

$\mathrm{C}$ atoms, the contribution to the AIS charge of these $\pi$ bond electrons is shared between the A sites of neighboring sheets. Since the $\mathrm{C}$ atom at the $\mathrm{B}$ site has no direct neighbor on the neighboring sheet the contribution goes entirely to this atom, resulting in the slightly negatively charged $\mathrm{C}$ atom. Charge neutrality results in a slightly positively charged $\mathrm{C}$ atom at the A site. Similar behavior was observed by Baranov and Kohout ${ }^{59}$ using the Bader approach. These authors, however, find a larger and opposite charge transfer, resulting in a charge of $+0.08 \mathrm{e}$ and $-0.08 \mathrm{e}$ on the $\mathrm{C}_{A}$ and $\mathrm{C}_{B}$ atoms respectively. This difference could originate from the different methods used.

The ceria compounds show the behavior expected with regard to equivalent/inequivalent atoms. The Hirshfeld-I values presented in Table IV are comparable with Bader charges presented in literature. Castleton et al. found for $\mathrm{CeO}_{2}$ Bader charges of $+2.3 \mathrm{e}$ and $-1.15 \mathrm{e}$ for $\mathrm{Ce}$ and O, respectively. ${ }^{60}$ The Mulliken atomic charges for Ce in $\mathrm{Ce}_{2} \mathrm{O}_{3}$ presented in literature appear strongly dependent on the functional used, varying from $+1.29 \mathrm{e}$ for PBE up to +2.157 e for Hartree-Fock. ${ }^{61,62}$ The lack of Hirshfeld-I values in the literature makes it difficult to make a true qualitative assessment of our obtained results. However, our results appear to show an overall qualitative agreement with the results obtained from other AIM methods. Table IV also shows there is a clear difference between the tri-and tetra-valent Ce ions, also the different configurations for the $\mathrm{O}$ ions show distinctly different charges. Looking at the relative atomic charges of the $\mathrm{Ce}$ and $\mathrm{O}$ atoms in $\mathrm{CeO}_{2}$ and $\mathrm{Ce}_{2} \mathrm{O}_{3}$ we find the same relative order as was found by Hay et al. ${ }^{61}$ and a difference in atomic charge for the Ce ions of comparable size. The different charges for the tri-and tetra-valent Ce ions might tempt one to consider these charges as indicators of the oxidation state if not the actual oxidation state of the atoms involved. As a result one could then assume that the same charge in a different configuration would be the result of the same oxidation state ( $c f$. concept of transferability). Looking at the charges of the $\mathrm{O}$ atoms in both $\mathrm{CeO}_{2}$ and $\mathrm{Ce}_{2} \mathrm{O}_{3}$ shows this is clearly not the case, since all $\mathrm{O}$ atoms formally have the same oxidation state, while the calculated Hirshfeld-I charges vary 0.2 electron. The Hirshfeld charges on the other hand show a much smaller variation of only 0.03 electron. At this point, it is important to stress that atomic charges do not, as opposed to what is often assumed, directly reveal the oxidation state, nor the valence of an atom. A Hirshfeld(-I) analysis, just like a Bader analysis, can only reveal atomic domains. The actual valence of an atom can be derived from the localization indices, which correspond in the simplest form to integrating twice the exchange-correlation density over the same atomic domain. ${ }^{63,64}$ Delocalization indices are obtained from double integration of the exchange correlation density over two different atomic domains. ${ }^{65}$ Such matrices have been less thoroughly explored in solid state calculations. $^{59}$

Another interesting point to note is that different spinconfigurations have little to no influence on the obtained charges. This is seen when comparing the FM and AF configurations of $\mathrm{Ce}_{2} \mathrm{O}_{3}$. This means that for generating the required system EDDs for $\mathrm{Ce}_{2} \mathrm{O}_{3}$ a non-spinpolarized calculation suffices for the study of the system. Note, however, that the single atom calculations used to generate the reference radial densities are spin-polarized.

\section{CONCLUSION}

We have presented an implementation of the HirshfeldI method specifically aimed at periodic systems, such as wires, surfaces, and bulk materials. Instead of calculating the electron densities at each point in space on the fly using the precalculated wave function of the system, we interpolate the electron density from a precalculated EDD on a dense spatial grid, speeding up the calculation of the density significantly. The use of such grids is possible because PBC allow for the use of a relatively small grid to describe the entire system accurately.

Unlike total energy calculations, the number of atoms involved can not be fully reduced to only those in the unit cell. Although, the populations only need to be calculated for the atoms in the unit cell, the Hirshfeld-I calculations require a large 'sphere of influence' containing a few thousand of atoms. By selecting only the grid points which contribute significantly to the calculations, the computational cost of the used multi-center integration grids can be substantially reduced.

We have shown that the uniform grids used to store both the atom and the system EDDs have an equal influence on the accuracy of the final Hirshfeld-I calculated populations, leading to the suggestion of building the library of atomic radial EDDs using as dense as possible grids. In addition, we have shown that both different 
atomic types and different chemical environments give rise to a different convergence behavior as function of the spherical integration grid.

The problems observed for the atomic radial EDDs of negatively charged ions are solved in a simple way, and we show that the introduced scaling of the distributions significantly improves the obtained results for the Hirshfeld-I charges. The resulting values for a benchmark set of 168 neutral molecules show very good agreement with the values obtained by a previous implementation of the Hirshfeld-I method aimed solely at molecular systems.

In the final section we have investigated some periodic systems to show the validity of our implementation. For each of these systems the expected behavior of the charges is observed. Because of their simplicity these systems are ideal test cases for Hirshfeld-I implementations for periodic systems.

\section{ACKNOWLEDGEMENT}

The research was financially supported by FWOVlaanderen, project $\mathrm{n}^{\circ} 3 \mathrm{G} 080209$. This work was carried out using the Stevin Supercomputer Infrastructure at Ghent University, funded by Ghent University, the Hercules Foundation and the Flemish Governmentdepartment EWI.
1 P. W. Ayers, J. Chem. Phys. 113, 10886 (2000).

2 R. F. W. Bader and C. F. Matta, J. Phys. Chem. A 108, 8385 (2004).

${ }^{3}$ C. F. Matta and R. F. W. Bader, J. Phys. Chem. A 110, 6365 (2006).

${ }^{4}$ R. G. Parr, P. W. Ayers, and R. F. Nalewajski, J. Phys. Chem. A 109, 3957 (2005).

${ }^{5}$ R. S. Mulliken, J. Chem. Phys. 3, 573 (1935).

6 R. S. Mulliken, J. Chem. Phys. 23, 1833 (1955).

7 F. L. Hirshfeld, Theor. Chim. Acta 44, 129 (1977).

8 P. Bultinck, Farad. Discuss. 135, 347 (2007), general Meeting on Chemical Concepts from Quantum Mechanics, Univ Manchester, Manchester, England, Sep 04-06, 2006.

9 P. Bultinck, C. Van Alsenoy, P. W. Ayers, and R. CarbóDorca, J. Chem. Phys. 126, 144111 (2007).

10 T. C. Lillestolen and R. J. Wheatley, Chem. Comm. pp. 5909-5911 (2008).

11 T. C. Lillestolen and R. J. Wheatley, J. Chem. Phys. 131, 144101 (2009).

12 T. A. Manz and D. S. Sholl, J. Chem. Theory Comp. 6, 2455 (2010).

13 R. F. W. Bader, Chem. Rev. 91, 893 (1991).

14 E. Francisco, A. M. Pendás, A. Costales, and M. GarcíaRevilla, Comp. Theor. Chem. 975, 2 (2011).

15 E. Francisco, A. Pendás, and M. Blanco, Theor. Chem. Acc. 128, 433 (2011).

16 A. M. Pendás, V. Luaña, L. Pueyo, E. Francisco, and P. Mori-Sánchez, J. Chem. Phys. 117, 1017 (2002).

17 X. Gonze, B. Amadon, P.-M. Anglade, J.-M. Beuken, F. Bottin, P. Boulanger, F. Bruneval, D. Caliste, R. Caracas, M. Ct, et al., Comp. Phys. Comm. 180, 2582 (2009).

18 M. D. Smith, S. M. Blau, K. B. Chang, M. Zeller, J. Schrier, and A. J. Norquist, Cryst. Growth Des. 11, 4213 (2011).

19 E. C. Glor, S. M. Blau, J. Yeon, M. Zeller, P. S. Halasyamani, J. Schrier, and A. J. Norquist, J. Solid State Chem. 184, 1445 (2011).

20 J. Schrier, ACS Applied Materials \& Interfaces 3, 4451 (2011)

21 O. Leenaerts, H. Peelaers, A. D. Hernández-Nieves, B. Partoens, and F. M. Peeters, Phys. Rev. B 82, 195436 (2010).

22 O. Leenaerts, B. Partoens, and F. M. Peeters, Appl. Phys. Lett. 92, 243125 (2008).
23 O. Leenaerts, B. Partoens, and F. M. Peeters, Phys. Rev. B 79, 235440 (2009).

${ }^{24}$ M. A. Spackman and D. Jayatilaka, CrystEngComm 11, 19 (2009).

25 M. A. Spackman and P. G. Byrom, Chem. Phys. Lett. 267, 215 (1997)

26 J. J. McKinnon, A. S. Mitchell, and M. A. Spackman, Chem.-Eur. J. 4, 2136 (1998).

27 J. J. McKinnon, M. A. Spackman, and A. S. Mitchell, Acta Crystallogr., Sect. B 60, 627 (2004).

28 S. K. Wolff, D. J. Grimwood, J. J. McKinnon, D. Jayatilaka, and M. A. Spackman, Crystal explorer 2.1 (2007).

29 T. Watanabe, T. A. Manz, and D. S. Sholl, J. Phys. Chem. C 115, 4824 (2011).

${ }^{30}$ G. W. Rogers and J. Z. Liu, J. Am. Chem. Soc. 133, 10858 (2011).

31 T. Verstraelen, S. V. Sukhomlinov, V. Van Speybroeck, M. Waroquier, and K. S. Smirnov, J. Phys. Chem. C 116, 490 (2012).

32 D. E. P. Vanpoucke, HIVE v2.1, http://users.ugent.be/ $\sim$ devpouck/hive_refman/index.html (2011).

33 P. E. Blöchl, Phys. Rev. B 50, 17953 (1994).

${ }^{34}$ G. Kresse and D. Joubert, Phys. Rev. B 59, 1758 (1999).

35 S. Van Damme, P. Bultinck, and S. Fias, J. Chem. Theory Comput. 5, 334 (2009).

36 P. Hohenberg and W. Kohn, Phys. Rev. 136, B864 (1964).

37 W. Kohn and L. J. Sham, Phys. Rev. 140, A1133 (1965).

38 J. C. Slater, The Self-Consistent Field for Molecular and Solids, vol. 4 of Quantum Theory of Molecular and Solids (McGraw-Hill, New York, 1974).

39 S. H. Vosko, L. Wilk, and M. Nusair, Can. J. Phys. 58, 1200 (1980).

${ }^{40}$ M. J. Frisch, G. W. Trucks, H. B. Schlegel, G. E. Scuseria, M. A. Robb, J. R. Cheeseman, J. A. Montgomery, Jr., T. Vreven, K. N. Kudin, J. C. Burant, et al., Gaussian 03, Revision E.01, Gaussian, Inc., Wallingford, CT, 2004.

41 A. D. Becke, J. Chem. Phys. 88, 2547 (1988).

${ }^{4}$ V. I. Lebedev and D. Laikov, Doklady Mathematics 59, 477 (1999).

43 E. R. Davidson and S. Chakravorty, Theor. Chim. Acta 83, 319 (1992).

44 E. Francisco, A. M. Pendás, M. A. Blanco, and A. Costales, J. Phys. Chem. A 111, 12146 (2007). 
45 P. W. Ayers, R. C. Morrison, and R. K. Roy, J. Chem. Phys. 116, 8731 (2002).

46 P. Bultinck, P. W. Ayers, S. Fias, K. Tiels, and C. Van Alsenoy, Chem. Phys. Lett. 444, 205 (2007).

47 D. Ghillemijn, P. Bultinck, D. Van Neck, and P. W. Ayers, J. Comput. Chem. 32, 1561 (2011).

${ }^{48}$ G. Voronoï, Journal für die Reine und Angewandte Mathematik 133, 97 (1907).

49 E. Wigner and F. Seitz, Phys. Rev. 43, 804 (1933).

${ }^{50}$ In theory, one might even use electron density data determined from X-ray crystallography. In that case however, there would be the problem of the choice of the atomic reference densities. One would have to use all electron atomic radial densities, which in itself is not really an issue. The choice of the functional, however, would be a bit of a wild card. Actual tests will have to be performed to investigate how significantly this choice influences the obtained results.

${ }^{51}$ In our setup we use the CHGCAR-file produced by VASP as input. Due to it's simple format this means that comparably formatted input can easily be generated for different solid state codes, making our setup independent of the used solid state/quantum chemical code.

${ }^{52}$ Note that for the DDEC-code no such corrections are mentioned for the similar c1 method. The effect of using such under-populated reference anions is shown by comparison of Fig. 5a to Fig. 5b or c.
53 R. G. Parr and L. J. Bartolotti, J. Phys. Chem. 87, 2810 (1983).

54 R. G. Parr and W. Yang, Density-Functional Theory of Atoms and Molecules, vol. 16 of International series of monographs on chemistry (Oxford Science Publications, Oxford, 1989).

55 J. P. Perdew, R. G. Parr, M. Levy, and J. L. Balduz, Phys. Rev. Lett. 49, 1691 (1982).

56 W. Yang, Y. Zhang, and P. W. Ayers, Phys. Rev. Lett. 84, 5172 (2000).

57 VASP allows for the generation of all electron core (AECCAR0) and valence (AECCAR2) densities.

58 K. Momma and F. Izumi, J. Appl. Cryst. 41, 653 (2008).

59 A. I. Baranov and M. Kohout, J. Comput. Chem. 32, 2064 (2011).

60 C. W. M. Castleton, J. Kullgren, and K. Hermansson, J. Chem. Phys. 127, 244704 (2007).

61 P. J. Hay, R. L. Martin, J. Uddin, and G. E. Scuseria, J. Chem. Phys. 125, 034712 (2006).

62 J. Yang and M. Dolg, Theor. Chem. Acc. 113, 212 (2005).

${ }^{63}$ R. F. W. Bader and M. E. Stephens, J. Am. Chem. Soc. 97, 7391 (1975).

${ }^{64}$ R. Ponec and D. L. Cooper, Faraday Discuss. 135, 31 (2007).

65 P. Bultinck, D. L. Cooper, and R. Ponec, J. Phys. Chem. A 114, 8754 (2010). 